\title{
Autophagy activation contributes to glutathione transferase Mu 1-mediated chemoresistance in hepatocellular carcinoma
}

\author{
XIU-TAO FU ${ }^{1 *}$, KANG SONG $^{1 *}$, JIAN ZHOU $^{1,2^{*}}$, YING-HONG SHI ${ }^{1}$, WEI-REN LIU ${ }^{1}$, MENG-XIN TIAN $^{1}$, \\ LEI JIN ${ }^{1}$, GUO-MING SHI ${ }^{1}$, QIANG GAO ${ }^{1}$, ZHEN-BIN DING ${ }^{1}$ and JIA FAN ${ }^{1,2}$ \\ ${ }^{1}$ Department of Liver Surgery, Zhongshan Hospital, Key Laboratory of Carcinogenesis and Cancer Invasion, \\ Ministry of Education; ${ }^{2}$ Department of Molecular Cell Biology, Institute of Biomedical Sciences, \\ Fudan University, Shanghai 200032, P.R. China
}

Received March 26, 2017; Accepted March 21, 2018

DOI: $10.3892 / \mathrm{ol} .2018 .8667$

\begin{abstract}
Glutathione transferase Mu 1 (GSTM1) induces cancer drug resistance by hydrolyzing cancer chemotherapeutics or activating the anti-apoptosis pathway. However, the chemoresistance-inducing mechanism of GSTM1 in hepatocellular carcinoma (HCC) remains unknown. In the present study, the expression of GSTM1 was examined in three HCC cell lines. Oxaliplatin and sorafenib were selected as chemotherapeutic agents. Small interfering RNA was used to decrease GSTM1 expression. Cell death was measured using MTT and annexin V/propidium iodide assays. Activation of autophagy was evaluated by green fluorescent protein-light chain 3 redistribution and analysis of autophagy-related 5 expression in MHCC97-H and Huh-7 cells. A stepwise increase in GSTM1 expression with increasing metastatic potential of HCC cell lines was revealed. Cell death induced by oxaliplatin and sorafenib was significantly increased following GSTM1-knockdown in MHCC97-H and Huh-7 cells. Activation of autophagy was significantly inhibited by silencing GSTM1 expression. The results of the present study suggest that GSTM1 may protect HCC cells against the effect of oxaliplatin treatment through activating autophagy. The present study provides a novel perspective on HCC drug-resistance.
\end{abstract}

Correspondence to: Dr Zhen-Bin Ding or Dr Jia Fan, Department of Liver Surgery, Zhongshan Hospital, Key Laboratory of Carcinogenesis and Cancer Invasion, Ministry of Education, Fudan University, 1609 Xietu Road, Shanghai 200032, P.R. China

E-mail: ding.zhenbin@zs-hospital.sh.cn

E-mail: fan.jia@zs-hospital.sh.cn

*Contributed equally

Key words: hepatocellular carcinoma, glutathione transferase Mu 1, autophagy, chemoresistance, oxaliplatin

\section{Introduction}

Hepatocellular carcinoma (HCC) is the second leading cause of cancer-associated mortality worldwide (1). Of patients diagnosed with liver cancer, $>70 \%$ are diagnosed too late for successful surgical treatment, and, of patients with HCC who undergo surgery, between 60 and $70 \%$ relapse within 5 years $(2,3)$. Therefore, there is an urgent requirement to improve antitumor drugs for advanced and recurrent HCC. Sorafenib, a multi-kinase inhibitor that prevents tumor cell proliferation by targeting the RAF proto-oncogene/mitogen-activated protein kinase signaling pathway, also inhibits the vascular endothelial growth factor receptor and platelet-derived growth factor receptor- $\beta$, thereby inhibiting angiogenesis (4). Sorafenib is the first line of treatment against unresectable HCC $(5,6)$. However, widespread sorafenib-resistance exists in patients with advanced HCC, which significantly decreases the therapeutic efficacy of the drug $(7,8)$. The third-generation platinum drug oxaliplatin has been widely used for the treatment of various types of cancer, including colon, gastric and metastatic liver cancer (9). Clinical trials of oxaliplatin in advanced HCC revealed moderate activity, but limited efficacy (10). The reason for this is partly intrinsic multidrug resistance and acquired drug resistance following chemotherapy (11). Therefore, elucidation of the mechanism of drug resistance is required to improve the prognosis of patients with HCC.

The glutathione transferases (GSTs) are a gene superfamily of phase II metabolic enzymes that detoxify free radicals derived from tobacco smoke, oxidative stress and carcinogens, including benzopyrene and other polycyclic aromatic hydrocarbons (12). A previous study by our group demonstrated that the GST Mu 1 (GSTM1)-null genotype may increase the risk of HCC (13). It is speculated that the expression of GSTM1 is associated with tumor suppression in HCC tumorigenesis. However, the GSTs are considered to be directly involved in the metabolic pathways of drug resistance (14). It was also demonstrated that GSTs were able to function in vivo and in vitro as endogenous repressors of the opening of a permeability transition pore complex, which contributed to chemotherapy-induced apoptosis (15). Thus, GSTs may protect tumor cells from chemotherapy drugs via metabolic or 
non-metabolic pathways. In the present study, the underlying molecular mechanism of GSTM1-mediated chemoresistance in HCC was investigated.

A previous study by our group demonstrated that autophagy defects during early stages of oncogenesis may contribute to the malignant differentiation and invasive phenotype of HCC (16). Furthermore, autophagy is able to protect HCC tumor cells against anti-neoplastic agent-induced apoptosis (17). In the present study, oxaliplatin and sorafenib were selected as chemotherapeutic agents, and the levels of GSTM1 were analyzed in HCC cell lines with various metastatic potentials. It was hypothesized that there would be an association between GSTM1 expression and autophagy following oxaliplatin treatment.

\section{Materials and methods}

Reagents. Oxaliplatin was purchased from Sigma-Aldrich; Merck KGaA (Darmstadt Germany). Sorafenib was synthesized at Bayer (Newbury, UK). Oxaliplatin and sorafenib were dissolved in Dulbecco's modified Eagle's medium (DMEM) containing $0.1 \%$ dimethylsulfoxide (Invitrogen; Thermo Fisher Scientific, Inc., Waltham, MA, USA).

Cell lines. The HCC cell lines HCCLM3 and MHCC97-H (established in Liver Cancer Institute of Zhongshan Hospital, Fudan University, Shanghai China) have different lung metastatic potentials and the same genetic background. The HCC cell line Huh-7 has low metastatic potential, and was purchased from the Institute of Biochemistry and Cell Biology (The Chinese Academy of Sciences, Shanghai, China). All cells were maintained in high-glucose DMEM supplemented with $10 \%$ heat-inactivated fetal bovine serum, 100 units $/ \mathrm{ml}$ penicillin and $100 \mathrm{mg} / \mathrm{ml}$ streptomycin. All cells were cultured at $37^{\circ} \mathrm{C}$ in a humidified incubator containing $5 \% \mathrm{CO}_{2}$.

MTT assay. The cell viability was assessed using an MTT cell proliferation assay kit (Trevigen Inc., Gaithersburg, MD, USA), according to the manufacturer's protocol. In total, $5 \times 10^{3}$ cells were plated in 96 -well plates, incubated for $24 \mathrm{~h}$ at $37^{\circ} \mathrm{C}$. After $24 \mathrm{~h}$ incubation and attachment, the cells were treated with $10 \mu \mathrm{mol} / 1$ oxaliplatin or $20 \mu \mathrm{mol} / 1$ sorafenib for an additional $12,24,36$, and $48 \mathrm{~h}$, respectively. Then, $20 \mu \mathrm{l}$ of MTT solution $(5 \mathrm{mg} / \mathrm{ml})$ was added to each well for and incubated for $4 \mathrm{~h}$ at $37^{\circ} \mathrm{C}$. The supernatant in each well was then gently aspirated, and $150 \mu \mathrm{l}$ dimethyl sulfoxide was added to each well to dissolve the crystals, and the plate was shaken on a horizontal shaker for $10 \mathrm{~min}$. The optical density (OD) at $570 \mathrm{~nm}$ were measured using a microplate reader, and the inhibition ratio was calculated using the following equation: Inhibition ratio $(\%)=(1-\mathrm{OD}$ value of the experimental group/OD value of the control group) $\mathrm{x} 100$.

Annexin V/propidium iodide (PI) assay. The number of apoptotic cells was determined using annexin V/PI staining (Annexin V, Alexa Fluor 555 conjugate; Thermo Fisher Scientific, Inc.), according to the manufacturer's protocol (18). Cells were analyzed using a flow cytometer, and data were analyzed using CellQuest software version 3.3 (BD Bioscience, Franklin Lakes, NJ, USA).
Western blot analysis. Protein extraction from the HCCLM3, MHCC97-H and Huh-7 cells was performed using a radioimmunoprecipitation assay buffer (Beyotime Institute of Biotechnology, Haimen, China) containing 1\% protease inhibitor. Total protein was measured using a bicinchoninic acid assay kit (Pierce; Thermo Fisher Scientific, Inc.) according to the manufacturer's protocol. A total of, $200 \mu \mathrm{g} / \mathrm{well}$ protein was loaded in 5\% acrylamide and separated by $10 \%$ SDS-PAGE and transferred onto polyvinylidene difluoride membranes. The membranes were washed in TBS 3 times and blocked in TBS with 0.05\% Tween-20 (ST825, Beyotime Institute of Biotechnology) containing 5\% non-fat dried milk for $1 \mathrm{~h}$ at room temperature, and the membrane was then incubated with primary antibodies against the following: GSTM1 (dilution, 1:1,000; cat. no. ab113432; Abcam, Cambridge, UK), autophagy related 5 (ATG5) (dilution, 1:1,000; cat. no. 12994; CST Biological Reagents Co., Ltd., Shanghai, China) and GAPDH (dilution, 1:10,000; cat. no. AP0063; Bioworld Technology, Inc., St. Louis Park, MN, USA) at $4^{\circ} \mathrm{C}$ overnight. The membranes were then incubated with a horseradish peroxidase-conjugated goat anti-rabbit secondary antibody (dilution, 1:10,000; cat. no. A16110; Thermo Fisher Scientific, Inc., Waltham, MA, USA) for $2 \mathrm{~h}$ at room temperature. The protein bands were visualized using enhanced chemiluminescence western blotting substrate (Pierce; Thermo Fisher Scientific, Inc.), and captured by ChemiDoc ${ }^{\mathrm{TM}} \mathrm{XRS}+$ system (Bio-Rad Laboratories, Hercules, CA, USA) and the densitometry of the protein bands were determined by ImageLab version 3.0 (Bio-Rad Laboratories).

RNA interference. High-performance purity grade small interfering RNA (siRNA; $>90 \%$ pure) against GSTM1 (GSTM1-siRNA) was obtained from OriGene Technologies, Inc. (cat. no. SR301988; Rockville, MD, USA). A non-silencing oligonucleotide sequence was used as a negative control (negative control siRNA, cat. no. SR30004; OriGene Technologies, Inc.). MHCC97-H and Huh-7 cells were seeded at density of $5 \times 10^{4}$ cells/well in 6-well plates and cultured in DMEM containing $10 \%$ FBS. At 1 day after seeding, cells were transfected with 100 pmol GSTM1-siRNA or negative siRNA using Lipofectamine ${ }^{\mathrm{TM}} 2000$ (Thermo Fisher Scientific, Inc.), according to the manufacturer's protocol. Cells were lysed $72 \mathrm{~h}$ after transfection, and protein was analyzed by western blotting.

Autophagy analysis. For the quantitative analysis of autophagy, $2 \times 10^{5}$ of MHCC97-H or Huh-7 cells were seeded in 6-well plates at $37^{\circ} \mathrm{C}$ and transfected with $4 \mu \mathrm{g}$ GFP-LC3 plasmid (concentration, $1 \mu \mathrm{g} / \mu \mathrm{l}$; Beyotime Institute of Biotechnology, Haimen, China) at $25^{\circ} \mathrm{C}$ using Lipofectamine 2000 (Invitrogen; Thermo Fisher Scientific, Inc.), according to the manufacturer's protocol for $24 \mathrm{~h}$ and then the cells were exposed to $10 \mu \mathrm{mol} / 1$ oxaliplatin for $12 \mathrm{~h}$ at $37^{\circ} \mathrm{C}$. Autophagy was assessed by green fluorescent protein (GFP)-light chain 3 (LC3) redistribution analysis. Redistribution of GFP-LC3 was assessed by determining the number of GFP-LC3-positive dots per transfected cell in three independent experiments using an inverted fluorescence microscope (magnification, x200; Nikon Corporation, Tokyo, Japan). In total, eight randomly selected fields, each containing 200 cells, were analyzed per well. 
Statistical analysis. All data are presented as the mean \pm standard deviation. An unpaired two-tailed Student's $\mathrm{t}$-test was used to compare the difference between two groups, and one-way analysis of variance was used to compare $\geq 3$ groups, followed by the Dunnett's post-hoc test. $\mathrm{P}<0.05$ was considered to indicate a statistically significant difference.

\section{Results}

GSTM1 expression in HCC cell lines. Western blotting revealed a $26-\mathrm{kDa}$ band, corresponding to membrane-bound GSTM1 protein in human HCC cell lines. Semi-quantitative analysis demonstrated that the GSTM1 protein expression level increased with the increasing metastatic potential of HCCLM3, MHCC97-H and Huh-7 cells $(\mathrm{P}<0.01)$ (Fig. 1).

Inhibition of GSTM1 promotes apoptosis following treatment with oxaliplatin. To investigate further the function of GSTM1 in chemotherapy-induced cell death, the expression of GSTM1 was inhibited using GSTM1-siRNA transfection of highly invasive MHCC97-H cells and low-invasive Huh-7 cells. Western blotting revealed that the protein expression level of GSTM1 was markedly inhibited following transfection, indicating that GSTM1 expression was successfully silenced by RNA interference (Fig. 2).

There was no difference in viability prior to oxaliplatin or sorafenib treatment. However, inhibition of GSTM1 expression in Huh-7 cells abolished the protective effect of GSTM1 and decreased cell viability by 6.7 and $11 \%$, respectively, following treatment with oxaliplatin for 24 and $48 \mathrm{~h}$, compared with control cells. Similarly, silencing GSTM1 expression in MHCC97-H cells markedly decreased cell viability following treatment with oxaliplatin for 24 and 48 h by 3.3 and $8.2 \%$, respectively, compared with control cells (Fig. 3A). Induction of apoptosis by oxaliplatin was further evaluated by annexin $\mathrm{V}$ staining. As presented in Fig. 3B, silencing of GSTM1 significantly increased the proportion of annexin V-positive MHCC97-H and Huh-7 cells following exposure to oxaliplatin. In addition, Huh-7 and MHCC97-H cells transfected with GSTM1-siRNA also demonstrated increased sensitivity to sorafenib (Fig. 3C).

Inhibition of GSTM1 protects against autophagy following oxaliplatin treatment. GFP-LC3 expression shifted from a diffuse cytoplasmic pattern to a punctate membranous pattern following exposure of MHCC97-H and Huh-7 cells to oxaliplatin for $12 \mathrm{~h}$, indicating the formation of autophagic vacuoles. Oxaliplatin-treated cells exhibited a significantly increased number of GFP-LC3-positive fluorescent autophagy vesicles per cell compared with untreated cells (Huh-7, $\mathrm{P}<0.05$; MHCC $97-\mathrm{H}, \mathrm{P}<0.01)$. GSTM1-siRNA transfection significantly decreased the number of fluorescent autophagy vesicles in oxaliplatin-treated cells, compared with negative control cells. No significant difference was identified between GSTM1-siRNA-transfected cells and negative control cells when not exposed to oxaliplatin (Fig. 4A and B). Western blotting revealed that the expression of ATG5 was markedly decreased in GSTM1-siRNA-transfected cells compared with negative control cells following oxaliplatin treatment (Fig. 5).

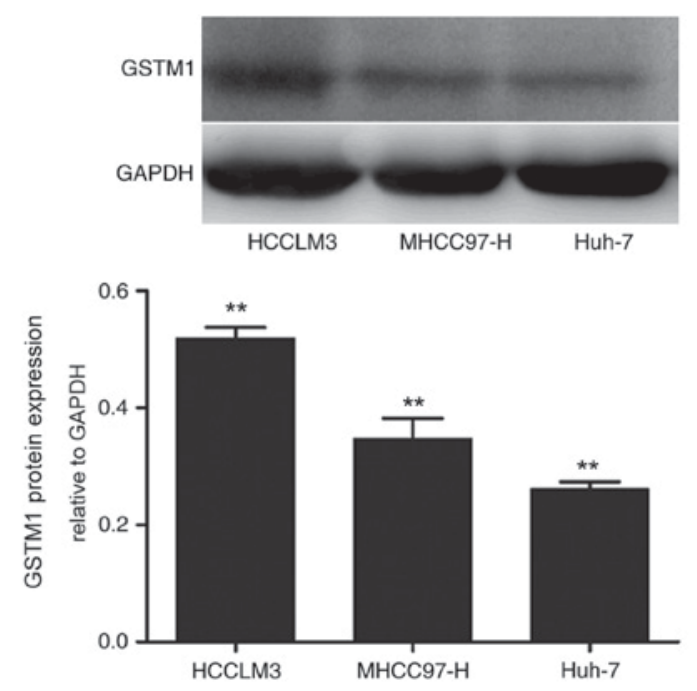

Figure 1. Expression of GSTM1 in HCC cell lines. The protein expression level of GSTM1 in HCCLM3, MHCC97-H and Huh-7 cells was examined by western blotting. Semi-quantitative analysis demonstrated that the GSTM1 protein expression level increased with the increasing metastatic potential of HCCLM3, MHCC97-H and Huh-7 cells. ${ }^{* *} \mathrm{P}<0.01$. GSTM1, glutathione transferase $\mathrm{Mu} 1$; HCC, hepatocellular carcinoma.
Huh-7

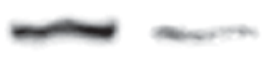

$\mathrm{MHCC} 97-\mathrm{H}$

GAPDH
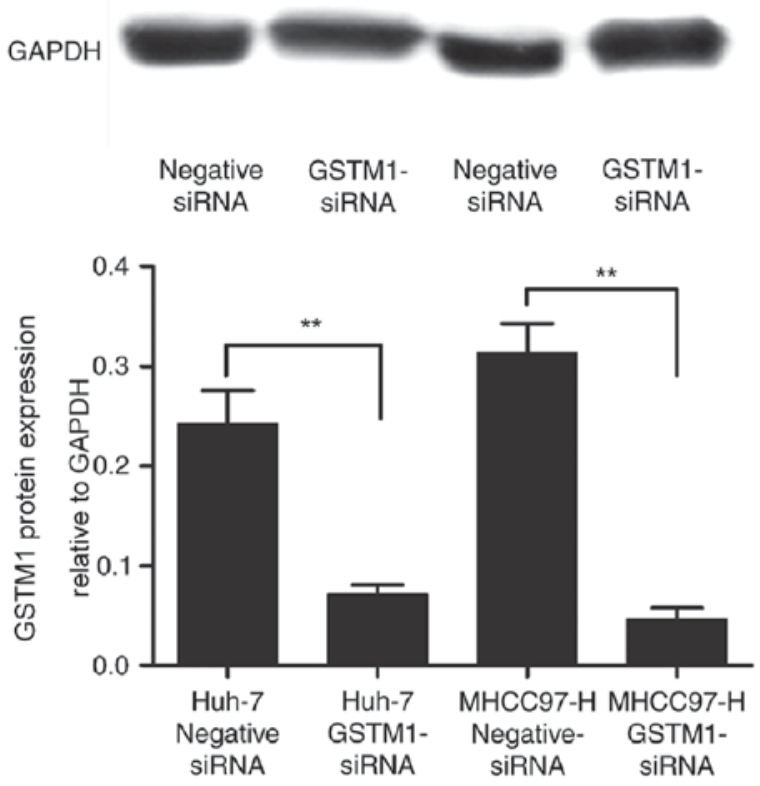

Figure 2. Silencing of GSTM1 expression in Huh-7 and MHCC97-H cells. Western blot analysis of the expression of GSTM1 in MHCC97-H and Huh-7 cells following GSTM1-siRNA transfection and it revealed that the protein expression level of GSTM1 was markedly inhibited following transfection, indicating that GSTM1 expression was successfully silenced by RNA interference. ${ }^{* *} \mathrm{P}<0.01$. GSTM, glutathione transferase Mu 1; negative siRNA, negative control small interfering RNA; GSTM1-siRNA, GSTM1-targeted small interfering RNA. 
A

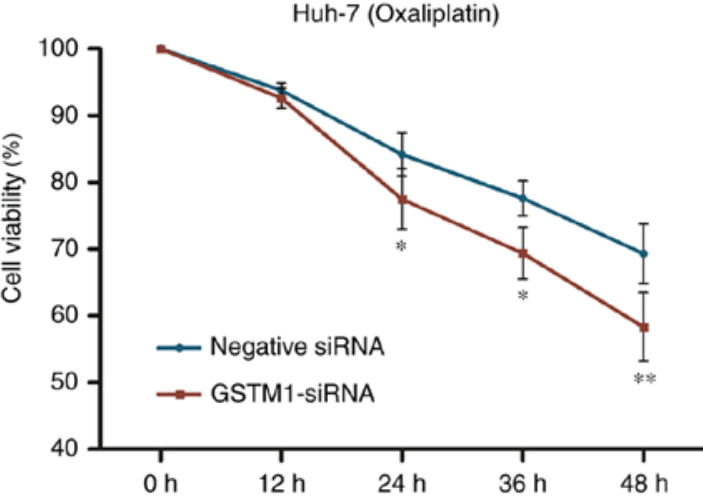

B

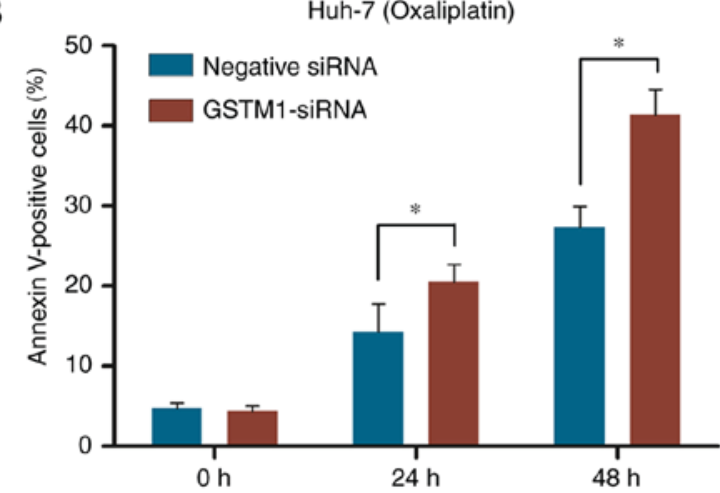

C

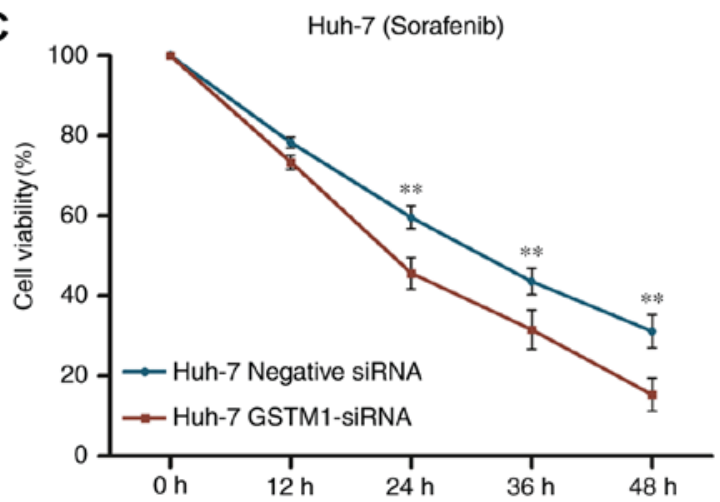

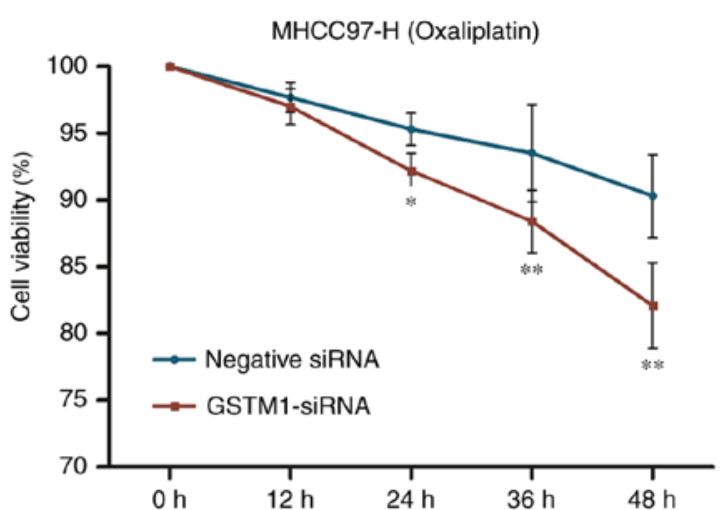

MHCC97-H (Oxaliplatin)
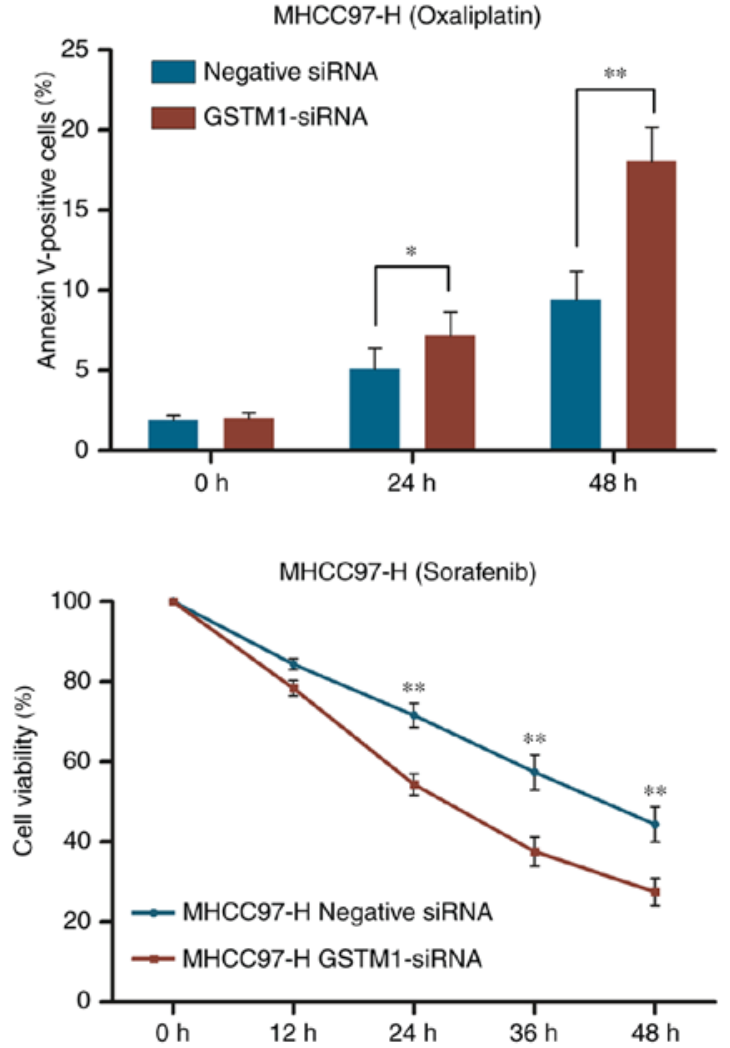

Figure 3. GSTM1-mediated apoptosis following treatment with oxaliplatin or sorafenib. (A) Huh-7 and MHCC97-H cells were treated with 10 $\mu$ mol/1 oxaliplatin for the indicated durations, and cell viability was determined using an MTT assay. Inhibition of GSTM1 expression in Huh-7 and MHCC97-H cells abolished the protective effect of GSTM1 to oxaliplatin. (B) Huh-7 and MHCC97-H cells were treated with $10 \mu$ mol/1 oxaliplatin following silencing of GSTM1. The percentage of dead cells was determined by annexin V staining. Silencing of GSTM1 significantly increased the proportion of annexin V-positive MHCC97-H and Huh-7 cells following exposure to oxaliplatin. (C) Huh-7 and MHCC97-H cells were treated with $20 \mu \mathrm{mol} / 1$ sorafenib for the indicated durations, and cell viability was determined using an MTT assay. Huh-7 and MHCC97-H cells transfected with GSTM1-siRNA demonstrated increased sensitivity to sorafenib. The data are presented as the mean \pm standard deviation of three independent experiments. ${ }^{*} \mathrm{P}<0.05,{ }^{* *} \mathrm{P}<0.01$. GSTM, glutathione transferase Mu 1 ; negative, negative control small interfering RNA; GSTM1-siRNA, GSTM1-targeted small interfering RNA.

\section{Discussion}

Resistance to anticancer agents is a major obstacle in the improvement of cancer therapy. It is widely accepted that GSTs participate in drug resistance processes by catalyzing the conjugation of glutathione (GSH) to drugs, including cisplatin, oxaliplatin, cyclophosphamide and doxorubicin $(19,20)$. GS-drug conjugates are hydrophilic and easily fluxed out of the cell through transporter proteins, which often contribute to the mechanism of drug resistance (21). The results of the present study indicate that the expression of GSTM1 was low in HCC cells with low metastatic potential, compared with in
HCC cells with high metastatic potential. It has been demonstrated previously that HCC cell lines with high metastatic potential are less sensitive to chemotherapy (22). Therefore, it is speculated that GSTM1 expression is associated with cancer drug resistance in $\mathrm{HCC}$ cells.

MHCC97-H cells exhibit high metastatic potential $(23,24)$, and it has been demonstrated that MHCC97-H cells exhibit increased resistance to chemotherapeutics compared with HCC cell lines with low metastatic potential (23-25). Similarly, previous studies have demonstrated that Huh-7 cells exhibit low metastatic potential and relatively weak chemoresistance $(26,27)$. In the present study, it was revealed 
A

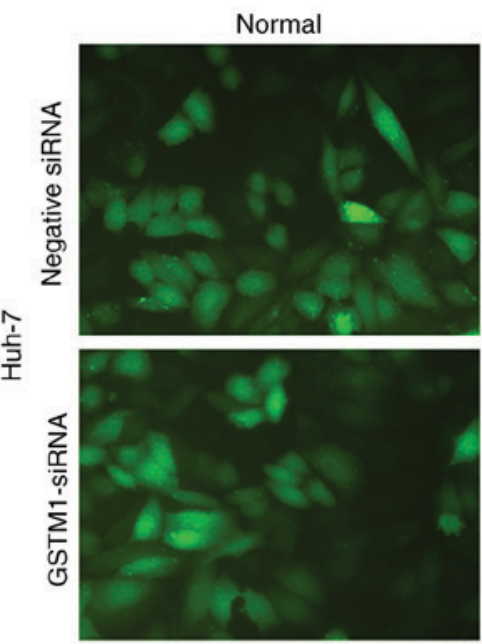

B

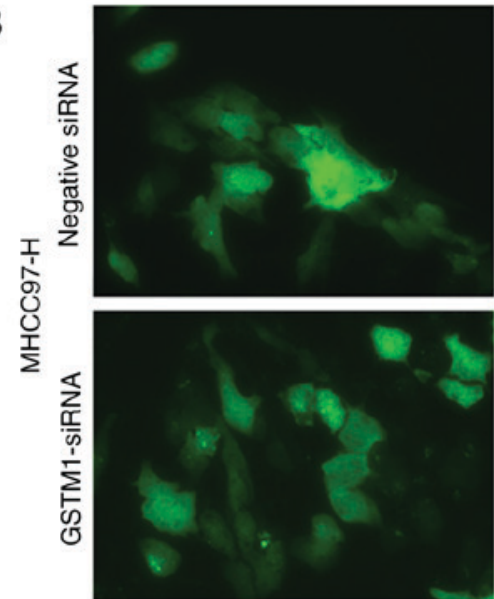

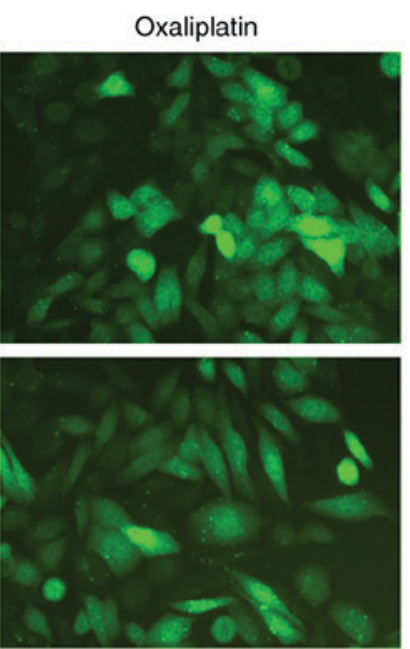
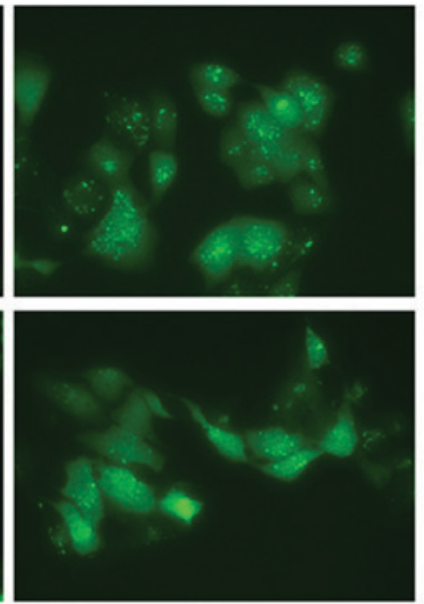

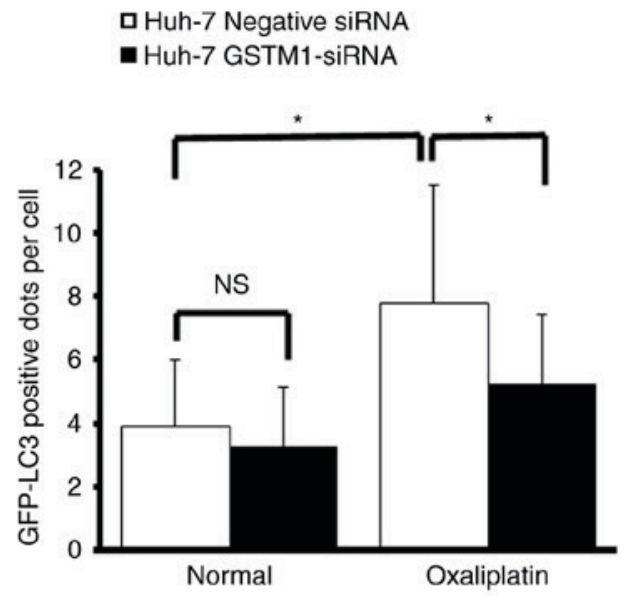

口MHCC97-H Negative SiRNA MHCC97-H GSTM1-SiRNA

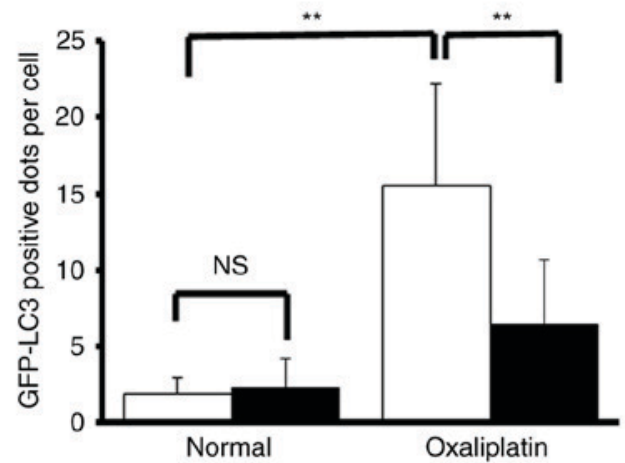

Figure 4. Silencing of GSTM1 expression protects against autophagy following oxaliplatin treatment. (A) Huh-7 and (B) MHCC97-H stably expressing the GFP-LC3 fusion protein were established. Following transfection with negative siRNA or GSTM1-siRNA, cells were exposed to $10 \mu \mathrm{mol} / 1$ oxaliplatin for $12 \mathrm{~h}$ and imaged using fluorescent microscopy. Magnification, x200. The number of GFP-LC3-positive dots per transfected cell were determined in three independent experiments. In total, eight random fields representing 200 cells were examined. The data are presented as the mean \pm standard deviation of three independent experiments. GSTM1-siRNA transfection significantly decreased the number of fluorescent autophagy vesicles in oxaliplatin-treated cells, compared with negative control cells. No significant difference was identified between GSTM1-siRNA-transfected cells and negative control cells when not exposed to oxaliplatin. ${ }^{*} \mathrm{P}<0.05,{ }^{* *} \mathrm{P}<0.01$. GSTM, glutathione transferase Mu 1; GFP, green fluorescent protein; LC3, light chain 3; negative siRNA, negative control small interfering RNA; GSTM1-siRNA, GSTM1-targeted small interfering RNA; NS, no significant difference.

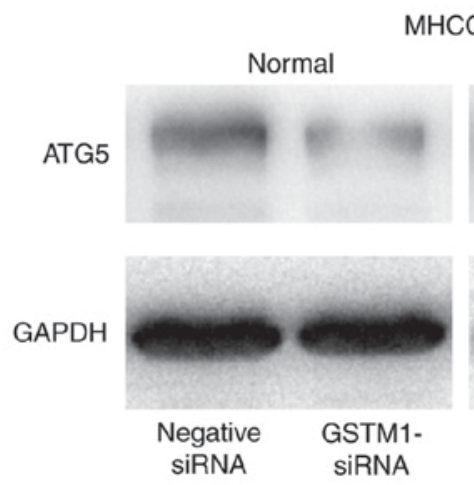

MHCC97-H
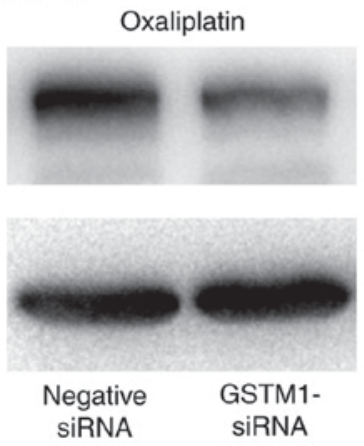

Huh-7

Normal
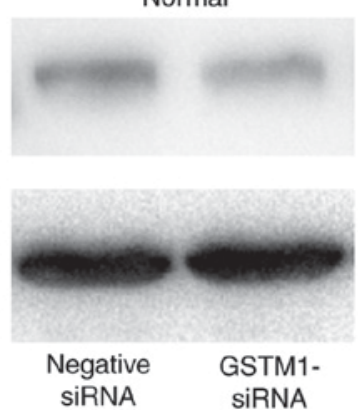

Oxaliplatin
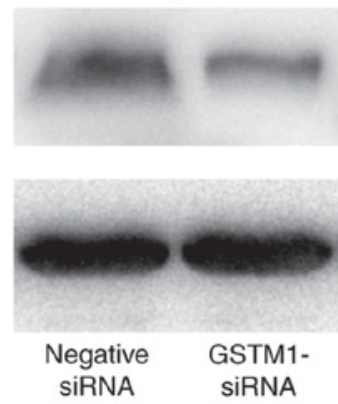

Figure 5. Western blot analysis of ATG5 protein expression in oxaliplatin-treated HCC cells. The protein expression level of ATG5 in Huh-7 and MHCC97-H was markedly decreased in GSTM1-siRNA-transfected cells compared with negative control cells following oxaliplatin treatment for $12 \mathrm{~h}$. ATG5, autophagy-related 5; HCC, hepatocellular carcinoma; negative siRNA, negative control small interfering RNA; GSTM1-siRNA, GSTM1-targeted small interfering RNA.

that knockdown of GSTM1 in MHCC97-H and Huh-7 cells increased resistance to oxaliplatin and sorafenib. Furthermore, our previous studies revealed that oxaliplatin and sorafenib could induce autophagy of HCC cells which contributed to 
chemo-resistance $(28,29)$, and it is reasonable to presume that this effect was associated with GSTM1-derived anti-apoptotic activity; however, it may not be associated with the general property of glutathione conjugation by GST.

Autophagy serves as a dynamic recycling system, which provides resources for cell homeostasis and repair (30). It has been demonstrated that autophagy is able to protect cancer cells against hypoxia, metabolic stress, detachment-induced anoikis and apoptosis or necrosis induced by antitumor therapy or other cell death stimuli (17,31-34). ATG5 forms a conjugate with autophagy-related 12 to function in autophagosome formation, and is considered to be a marker of autophagy (29,35). To directly determine the level of autophagy in MHCC97-H and Huh-7 cells following treatment with oxaliplatin, GFP-LC3 redistribution and the expression of ATG5 were analyzed. The increased number of fluorescent autophagy vesicles per cell in oxaliplatin-treated cells compared with in untreated cells indicated that oxaliplatin was able to induce autophagy in HCC cells. Silencing of GSTM1 expression using siRNA allowed the investigation of the association between GSTM1 and autophagy in HCC cells. The results revealed that oxaliplatin-induced autophagy could be downregulated by silencing GSTM1. It can be hypothesized that GSTM1 may affect autophagic activity in HCC cells. To the best of our knowledge, the present study is the first to investigate the GSTM1-mediated chemoresistance via autophagy in HCC. However, the molecular mechanism underlying the effect of GSTM1 on autophagy regulation remains unknown, and future research should focus on its elucidation.

A previous study revealed that activation of autophagy in HCC could contribute to the tolerance of oxaliplatin via regulation of the level of reactive oxygen species (ROS) (28). It has been demonstrated that GSTs serve an important function in cellular protection against oxidative stress in the respiratory tract via inhibition of the endogenous production of ROS, which is increased by exposure to sulfur mustard (36). Thus, it is speculated that regulation of ROS levels may involve GSTM1 and autophagic activity.

There are limitations to the present study. Overexpression of the gene of interest, GSTM1, may be more effective than gene knockdown. Thus, future studies will investigate the effect of overexpression of GSTM1 in HCC cell lines, and aim to identify a potential chemoresistance target in vivo. Furthermore, the present study would be improved by including tissues collected from patients with HCC exhibiting drug resistance.

In summary, GSTM1 may protect HCC cells against oxaliplatin treatment through activating autophagy. This provides a novel perspective to the investigation of drug resistance of HCC.

\section{Acknowledgements}

The authors would like to thank Dr Yan Zhao (Liver Cancer Institute of Zhongshan Hospital, Fudan University, Shanghai China) for her assistance with cell culture.

\section{Funding}

The present study was supported by the National Natural Science Fund of China (grant nos. 81472219 and 81602037).

\section{Availability of data and materials}

he datasets used and/or analyzed during the current study are available from the corresponding author on reasonable request.

\section{Authors' contributions}

$\mathrm{XF}, \mathrm{ZD}$ and JF designed the study and performed the literature review, supervised statistical analysis and interpretation of data and drafted the manuscript; KS, JZ, YS GS and QG contributed to the study design and drafting of the manuscript. WL, MT and LJ performed the molecular biological experiment. All authors critically revised the manuscript for intellectual and significant contents and approved the final manuscript for submission.

\section{Ethics approval and consent to participate}

Not applicable.

\section{Consent for publication}

Not applicable.

\section{Competing interests}

The authors declare that they have no competing interests.

\section{References}

1. El-Serag HB: Epidemiology of viral hepatitis and hepatocellular carcinoma. Gastroenterology 142: 1264-1273 e1261, 2012.

2. Hatzaras I, Bischof DA, Fahy B, Cosgrove D and Pawlik TM: Treatment options and surveillance strategies after therapy for hepatocellular carcinoma. Ann Surg Oncol 21: 758-766, 2014.

3. Chen MF, Hwang TL, Jeng LB, Wang CS, Jan YY and Chen SC: Postoperative recurrence of hepatocellular carcinoma. Two hundred five consecutive patients who underwent hepatic resection in 15 years. Arch Surg 129: 738-742, 1994

4. Wilhelm SM, Carter C, Tang L, Wilkie D, McNabola A, Rong H, Chen C, Zhang X, Vincent P, McHugh M, et al: BAY 43-9006 exhibits broad spectrum oral antitumor activity and targets the RAF/MEK/ERK pathway and receptor tyrosine kinases involved in tumor progression and angiogenesis. Cancer Res 64: 7099-7109, 2004.

5. Chao Y, Chung YH, Han G, Yoon JH, Yang J, Wang J, Shao GL, Kim BI and Lee TY: The combination of transcatheter arterial chemoembolization and sorafenib is well tolerated and effective in Asian patients with hepatocellular carcinoma: Final results of the START trial. Int J Cancer 136: 1458-1467, 2015.

6. Choi GH, Shim JH, Kim MJ, Ryu MH, Ryoo BY, Kang YK, Shin YM, Kim KM, Lim YS and Lee HC: Sorafenib alone versus sorafenib combined with transarterial chemoembolization for advanced-stage hepatocellular carcinoma: Results of propensity score analyses. Radiology 269: 603-611, 2013.

7. Hua L, Hu B, Yan D, Liu J, Shen Y, Zhao F, Shen C, Chen B and Cui X: Upregulated expression of Nucleostemin/GNL3 is associated with poor prognosis and Sorafenib Resistance in Hepatocellular Carcinoma. Pathol Res Pract 213: 688-697, 2017.

8. Zhu YJ, Zheng B, Wang HY and Chen L: New knowledge of the mechanisms of sorafenib resistance in liver cancer. Acta Pharmacol Sin 38: 614-622, 2017.

9. Qin S, Bai Y, Lim HY, Thongprasert S, Chao Y, Fan J, Yang TS, Bhudhisawasdi V, Kang WK, Zhou Y, et al: Randomized, multicenter, open-label study of oxaliplatin plus fluorouracil/leucovorin versus doxorubicin as palliative chemotherapy in patients with advanced hepatocellular carcinoma from Asia. J Clin Oncol 31: 3501-3508, 2013 
10. Zaanan A, Williet N, Hebbar M, Dabakuyo TS, Fartoux L, Mansourbakht T, Dubreuil O, Rosmorduc O, Cattan S, Bonnetain $\mathrm{F}$, et al: Gemcitabine plus oxaliplatin in advanced hepatocellular carcinoma: A large multicenter AGEO study. J Hepatol 58: 81-88, 2013.

11. Gobel T, Blondin D, Kolligs F, Bolke E and Erhardt A: Current therapy of hepatocellular carcinoma with special consideration of new and multimodal treatment concepts. Dtsch Med Wochenschr 138: 1425-1430, 2013 (In German).

12. Yang JX, Luo Y, Qiu HM and Tang WX: Characterization and resistance mechanisms of cisplatin-resistant human hepatocellular carcinoma cell line. Saudi Med J 30: 35-40, 2009.

13. Song K, Yi J, Shen X and Cai Y: Genetic polymorphisms of glutathione S-transferase genes GSTM1, GSTT1 and risk of hepatocellular carcinoma. PLoS One 7: e48924, 2012.

14. Di Pietro G, Magno LA and Rios-Santos F: Glutathione S-transferases: An overview in cancer research. Expert Opin Drug Metab Toxicol 6: 153-170, 2010.

15. Verrier F, Deniaud A, Lebras M, Métivier D, Kroemer G, Mignotte B, Jan G and Brenner C: Dynamic evolution of the adenine nucleotide translocase interactome during chemotherapy-induced apoptosis. Oncogene 23: 8049-8064, 2004.

16. Ding ZB, Shi YH, Zhou J, Qiu SJ, Xu Y, Dai Z, Shi GM, Wang XY, Ke AW, Wu B and Fan J: Association of autophagy defect with a malignant phenotype and poor prognosis of hepatocellular carcinoma. Cancer Res 68: 9167-9175, 2008.

17. Jin S and White E: Role of autophagy in cancer: Management of metabolic stress. Autophagy 3: 28-31, 2007.

18. King FW, Fong S, Griffin C, Shoemaker M, Staub R, Zhang YL, Cohen I and Shtivelman E: Timosaponin AIII is preferentially cytotoxic to tumor cells through inhibition of mTOR and induction of ER stress. PLoS One 4: e7283, 2009.

19. Tew KD: Glutathione-associated enzymes in anticancer drug resistance. Cancer Res 54: 4313-4320, 1994.

20. Ang WH, Khalaila I, Allardyce CS, Juillerat-Jeanneret L and Dyson PJ: Rational design of platinum(IV) compounds to overcome glutathione-S-transferase mediated drug resistance. J Am Chem Soc 127: 1382-1383, 2005.

21. Townsend DM and Tew KD: The role of glutathione-S-transferase in anti-cancer drug resistance. Oncogene 22: 7369-7375, 2003.

22. Yang T, Zheng ZM, Li XN, Li ZF, Wang Y, Geng YF, Bai L and Zhang XB: MiR-223 modulates multidrug resistance via downregulation of ABCB1 in hepatocellular carcinoma cells. Exp Biol Med (Maywood) 238: 1024-1032, 2013.

23. Zhao N, Wang R, Zhou L, Zhu Y, Gong J and Zhuang SM: MicroRNA-26b suppresses the NF- $\kappa$ B signaling and enhances the chemosensitivity of hepatocellular carcinoma cells by targeting TAK1 and TAB3. Mol Cancer 13: 35, 2014.

24. Zhou Z, Deng H, Yan W, Luo M, Tu W, Xia Y, He J, Han P, Fu Y and Tian D: AEG-1 promotes anoikis resistance and orientation chemotaxis in hepatocellular carcinoma cells. PLoS One 9: e100372, 2014
25. Qu Z, Wu J, Wu J, Luo D, Jiang C and Ding Y: Exosomes derived from HCC cells induce sorafenib resistance in hepatocellular carcinoma both in vivo and in vitro. J Exp Clin Cancer Res 35: 159,2016

26. Xu X, Liu RF, Zhang X, Huang LY, Chen F, Fei QL and Han ZG: DLK1 as a potential target against cancer stem/progenitor cells of hepatocellular carcinoma. Mol Cancer Ther 11: 629-638, 2012

27. Chen X, Lingala S, Khoobyari S, Nolta J, Zern MA and Wu J: Epithelial mesenchymal transition and hedgehog signaling activation are associated with chemoresistance and invasion of hepatoma subpopulations. J Hepatol 55: 838-845, 2011.

28. Ding ZB, Hui B, Shi YH, Zhou J, Peng YF, Gu CY, Yang H, Shi GM, Ke AW, Wang XY, et al: Autophagy activation in hepatocellular carcinoma contributes to the tolerance of oxaliplatin via reactive oxygen species modulation. Clin Cancer Res 17: 6229-6238, 2011.

29. Shi YH, Ding ZB, Zhou J, Hui B, Shi GM, Ke AW, Wang XY, Dai Z, Peng YF, Gu CY, et al: Targeting autophagy enhances sorafenib lethality for hepatocellular carcinoma via ER stress-related apoptosis. Autophagy 7: 1159-1172, 2011.

30. Mizushima N and Komatsu M: Autophagy: Renovation of cells and tissues. Cell 147: 728-741, 2011.

31. Moreau K, Luo S and Rubinsztein DC: Cytoprotective roles for autophagy. Curr Opin Cell Biol 22: 206-211, 2010.

32. Li J, Hou N, Faried A, Tsutsumi S, Takeuchi T and Kuwano H: Inhibition of autophagy by 3-MA enhances the effect of 5 -FU-induced apoptosis in colon cancer cells. Ann Surg Oncol 16: 761-771,2009.

33. Amaravadi RK, Yu D, Lum JJ, Bui T, Christophorou MA, Evan GI, Thomas-Tikhonenko A and Thompson CB: Autophagy inhibition enhances therapy-induced apoptosis in a Myc-induced model of lymphoma. J Clin Invest 117: 326-336, 2007.

34. Tian F, Deguchi K, Yamashita T, Ohta Y, Morimoto N, Shang J, Zhang X, Liu N, Ikeda Y, Matsuura T and Abe K: In vivo imaging of autophagy in a mouse stroke model. Autophagy 6: 1107-1114, 2010.

35. Klionsky DJ, Abdalla FC, Abeliovich H, Abraham RT, Acevedo-Arozena A, Adeli K, Agholme L, Agnello M, Agostinis P, Aguirre-Ghiso JA, et al: Guidelines for the use and interpretation of assays for monitoring autophagy. Autophagy 8: 445-544, 2012.

36. Nourani MR, Azimzadeh S, Ghanei M and Imani Fooladi AA: Expression of glutathione S-transferase variants in human airway wall after long-term response to sulfur mustard. J Recept Signal Transduct Res 34: 125-130, 2013

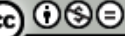

This work is licensed under a Creative Commons Attribution-NonCommercial-NoDerivatives 4.0 International (CC BY-NC-ND 4.0) License. 